\title{
Synthesis and Structure of 1,4-Dipiperazino Benzenes - Chiral Terphenyl-type Peptide Helix Mimetics
}

\section{Supporting Information}

\author{
Page
}

Synthesis of new compounds

S-2

CD-Measurements

S-13

Concentration dependent NMR

S-14

$\mathrm{X}$-ray diffraction structure of compound $9 \mathrm{a}$

S-15

Copies of ${ }^{1} \mathrm{H}$ - and ${ }^{13} \mathrm{C}$ NMR

S-16 


\section{Experimental section}

General: Melting points were determined on a melting point apparatus and are uncorrected. Specific rotations were measured on a polarimeter using a $10 \mathrm{~cm}$ cell. NMR spectra were recorded in $\mathrm{CDCl}_{3}$ at $300 \mathrm{MHz}\left({ }^{1} \mathrm{H}\right)$ or $75 \mathrm{MHz}\left({ }^{13} \mathrm{C}\right)$ unless stated otherwise. Structural assignments are based on DEPT and COSY experiments where applicable. The multiplicity of the carbon atoms are given as $(+)=\mathrm{CH}, \mathrm{CH}_{3}$ or $(-)=\mathrm{CH}_{2}$ and $\left(\mathrm{C}_{\text {quat }}\right)$ for quaternary carbon atoms. Analytical TLC plates (silica gel $\left.60 \mathrm{~F}_{254}\right)$ and silica gel 60 (70-230 or 230-400 mesh) for column chromatography (CC) were purchased from ACROS. Spots were visualized by UV light and/or staining with phosphomolybdate or ninhydrin, both in ethanol. $\mathrm{DMF}$, toluene, and $\mathrm{MeOH}$ were dried by standard procedures and stored over molecular sieves or Na. PE means petrol ether with a boiling range of $70-90^{\circ} \mathrm{C}$. All other solvents and chemicals were of reagent grade and used without further purification.

\section{(S)-Methyl 2-(2-chloroacetamido)propanoate (2a):}

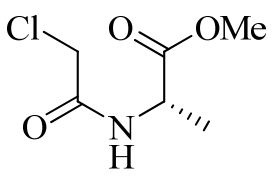

To a suspension of L-alanine $(26.7 \mathrm{~g}, 0.30 \mathrm{~mol})$ in $\mathrm{CH}_{3} \mathrm{OH}(200 \mathrm{~mL})$ cooled in an ice-salt bath, $\mathrm{SOCl}_{2}(87.5 \mathrm{~mL}, 1.2 \mathrm{~mol})$ was added drop-wise while stirring. After the addition the resulting mixture was stirred for an additional $6 \mathrm{~h}$ at $\mathrm{rt}$. The clear solution was concentrated to dryness, and without any further purification, the solid was dissolved in water $(120 \mathrm{~mL})$ and cooled in ice-salt bath. To the solution, $\mathrm{NaHCO}_{3}(60 \mathrm{~g}, 0.71 \mathrm{~mol})$ was added in one portion, and then the solution of chloroacetyl chloride $(23.8 \mathrm{~mL}, 0.3$ mol) in benzene $(100 \mathrm{~mL})$ was added dropwise. After the addition, the reaction mixture was stirred for an additional $3 \mathrm{~h}$ at $\mathrm{rt}$. The aqueous layer was extracted twice with benzene $(100 \mathrm{~mL})$, and the combined organic phases were dried over anhydrous $\mathrm{MgSO}_{4}$. Subsequent filtration and removal of organic solvent in vacuo gave a crude product 
(47.95 g, 89\%), which was purified by column chromatography on silica gel (3:1, PE: EtOAc) to give $43.0 \mathrm{~g}(81 \%)$ of product as a colorless oil. $[\alpha]_{\mathrm{D}}=-8.70^{\circ}\left(\mathrm{C}=2, \mathrm{CHCl}_{3}\right)$. ${ }^{1} \mathrm{H}-\mathrm{NMR}\left(\mathrm{CDCl}_{3}\right): \delta=1.45(\mathrm{~d}, \mathrm{~J}=7.1 \mathrm{~Hz}, 3 \mathrm{H}), 3.77(\mathrm{~s}, 3 \mathrm{H}), 4.06(\mathrm{~s}, 2 \mathrm{H}), 4.60(\mathrm{~m}, 1 \mathrm{H})$, $7.12(\mathrm{~b} \mathrm{~s}, 1 \mathrm{H})$.

(S)-Methyl 2-(2-chloroacetamido)-4-methylpentanoate (2b):<smiles>COC(=O)C(CC(C)C)NC(=O)CCl</smiles>

The compound was prepared by the same procedure described above. Yield: $78 \%$, colorless oil. $[\alpha]_{\mathrm{D}}=+6.60^{\circ}\left(\mathrm{C}=2, \mathrm{CHCl}_{3}\right)$.

${ }^{1} \mathrm{H}-\mathrm{NMR}\left(\mathrm{CDCl}_{3}\right): \delta=0.95(\mathrm{~d}, \mathrm{~J}=7.1 \mathrm{~Hz}, 6 \mathrm{H}), 1.57-1.78(\mathrm{~m}, 3 \mathrm{H}), 3.77(\mathrm{~s}, 3 \mathrm{H}), 4.06(\mathrm{~s}$, 2H), $4.60(\mathrm{~m}, 1 \mathrm{H}), 7.01(\mathrm{bs}, 1 \mathrm{H})$.

(S)-Methyl 2-(2-chloroacetamido)-3-phenylpropanoate (2c):<smiles>COC(=O)C(Cc1ccccc1)NC(=O)CCl</smiles>

The compound was prepared by the same procedure described above. Yield: $80 \%$, colorless oil. $[\alpha]_{\mathrm{D}}=+11.40^{\circ}\left(\mathrm{C}=2, \mathrm{CHCl}_{3}\right)$

${ }^{1} \mathrm{H}-\mathrm{NMR}\left(\mathrm{CDCl}_{3}\right): \delta=3.13-3.18(\mathrm{dd}, \mathrm{J}=3.9 \mathrm{~Hz}, \mathrm{~J}=3.9 \mathrm{~Hz}, 2 \mathrm{H}), 3.77(\mathrm{~s}, 3 \mathrm{H}), 4.01$ (s, 2H), 4.83-4.91 (m, 1H), 6.94-7.02 (bs, 1H), 7.09-7.34 (m, 5H).

${ }^{13} \mathrm{C}-\mathrm{NMR}\left(\mathrm{CDCl}_{3}\right): \delta=37.8(-), 42.4(-), 52.5(+), 53.4(+), 127.4(+), 128.7(+), 129.24$ $(+), 135.0(+), 135.4(+), 165.6\left(\mathrm{C}_{\text {quat }}\right), 171.3\left(\mathrm{C}_{\text {quat }}\right)$.

(S)-1-Benzyl-3-methylpiperazine-2, 5-dione (3a): 


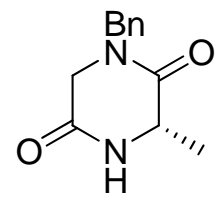

A solution of benzylamine ( $24.1 \mathrm{~mL}, 0.22 \mathrm{~mol})$ in $\mathrm{CH}_{3} \mathrm{OH}(180 \mathrm{~mL})$ was added dropwise over $1.5 \mathrm{~h}$ to a solution of compound $2 \mathrm{a}(32.8 \mathrm{~g}, 0.183 \mathrm{~mol})$ and TEA $(73.2 \mathrm{~mL}, 0.55$ mol) in $\mathrm{CH}_{3} \mathrm{OH}(180 \mathrm{~mL})$ and refluxed for $20 \mathrm{~h}$, the pale yellow solution was cooled to rt and concentrated, and the residue was dissolved in $150 \mathrm{~mL} \mathrm{CH}_{2} \mathrm{Cl}_{2}$. The organic phase was washed with $100 \mathrm{~mL}$ of $5 \%$ aqueous citric acid, $100 \mathrm{~mL}$ of saturated aqueous $\mathrm{NaHCO}_{3}$, and $100 \mathrm{~mL}$ of brine, and dried over $\mathrm{MgSO}_{4}$. Subsequent filtration and removal of organic solvent in vacuo gave a pale yellow solid, which was recrystallized from toluene to give $31.1 \mathrm{~g}(78 \%)$ of compound $3 \mathrm{a}$ as a white solid. Mp $138-140^{\circ} \mathrm{C} ;[\alpha]_{\mathrm{D}}=-$ $8.80^{\circ}\left(\mathrm{c}=2, \mathrm{CHCl}_{3}\right),\left\{\right.$ lit. $\left.^{1}[\alpha]_{\mathrm{D}}=-8.85^{\circ}\left(\mathrm{C}=2, \mathrm{CHCl}_{3}\right)\right\}$.

${ }^{1} \mathrm{H}-\mathrm{NMR}\left(\mathrm{CDCl}_{3}\right): \delta=1.53(\mathrm{~d}, \mathrm{~J}=7.0 \mathrm{~Hz}, 3 \mathrm{H}), 3.83(\mathrm{~s}, 2 \mathrm{H}), 4.16(\mathrm{~m}, 1 \mathrm{H}), 4.60(\mathrm{~s}, 2 \mathrm{H})$, 7.23-7.39 (m, 5H).

\section{(S)-1-Benzyl-3-isobutylpiperazine-2, 5-dione (3b):}

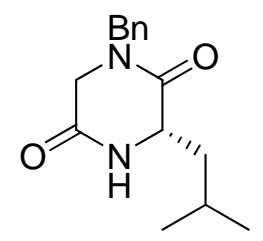

The compound was prepared by the same procedure described above. Yield: 70\%, white crystalline solid. $[\alpha]_{\mathrm{D}}=+6.40^{\circ}\left(\mathrm{C}=2, \mathrm{CHCl}_{3}\right)$.

${ }^{1} \mathrm{H}-\mathrm{NMR}\left(\mathrm{CDCl}_{3}\right): \delta=0.96(\mathrm{~d}, \mathrm{~J}=6.3 \mathrm{~Hz}, 3 \mathrm{H}), 0.98(\mathrm{~d}, \mathrm{~J}=6.3 \mathrm{~Hz}, 3 \mathrm{H}), 1.57-1.86(\mathrm{~m}$, $3 \mathrm{H}), 3.83(\mathrm{~m}, 2 \mathrm{H}), 4.16(\mathrm{~m}, 1 \mathrm{H}), 4.63(\mathrm{~d}, \mathrm{~J}=14.8 \mathrm{~Hz}, 1 \mathrm{H}), 4.64(\mathrm{~d}, \mathrm{~J}=14.8 \mathrm{~Hz}, 1 \mathrm{H})$, 7.20-7.39 (m, 5H).

(S)-1, 3-Dibenzylpiperazine-2, 5-dione (3c): 


\section{S-5}<smiles>O=C1CNC(=O)[C@H](Cc2ccccc2)N1</smiles>

The compound was prepared by the same procedure described above. Yield: $65 \%$, white crystalline solid. $[\alpha]_{\mathrm{D}}=+11.80^{\circ}\left(\mathrm{C}=2, \mathrm{CHCl}_{3}\right)$,

${ }^{1} \mathrm{H}-\mathrm{NMR}\left(\mathrm{CDCl}_{3}\right): \delta=2.89(\mathrm{~d}, \mathrm{~J}=17.6 \mathrm{~Hz}, 1 \mathrm{H}), 3.12(\mathrm{~d}, \mathrm{~J}=4.5 \mathrm{~Hz}, 1 \mathrm{H}), 3.15(\mathrm{~d}, \mathrm{~J}=4.5$ $\mathrm{Hz}, 1 \mathrm{H}), 3.23(\mathrm{~d}, \mathrm{~J}=6.2 \mathrm{~Hz}, 1 \mathrm{H}), 3.25(\mathrm{~d}, \mathrm{~J}=6.2 \mathrm{~Hz}, 1 \mathrm{H}), 3.48(\mathrm{~d}, \mathrm{~J}=17.6 \mathrm{~Hz}, 1 \mathrm{H}), 4.40$ (m, 1H), 4.60 (s, 2H), 7.13-7.39 (m, 10H).

${ }^{13} \mathrm{C}-\mathrm{NMR}\left(\mathrm{CDCl}_{3}\right): \delta=40.9(-), 48.4(-), 49.7(-), 56.5(+), 127.5(+), 128.1(+), 128.7$ $(+), 128.9(+), 130.1(+), 134.8\left(\mathrm{C}_{\text {quat }}\right), 134.9\left(\mathrm{C}_{\text {quat }}\right), 165.3\left(\mathrm{C}_{\text {quat }}\right), 166.3\left(\mathrm{C}_{\text {quat }}\right)$. MS $\left[\mathrm{CI}-\mathrm{MS} ; \mathrm{NH}_{3}\right]=312.2\left[{\mathrm{M}-\mathrm{NH}_{4}}^{+}\right](100), 295.1\left[\mathrm{M}-\mathrm{H}^{+}\right](20)$.

\section{(S)-1-Benzyl-3-methylpiperazine (4a):}<smiles>C[C@@H]1C[NH2+]CCN1</smiles>

A solution of diketopiperazine, 3a $(11.5 \mathrm{mmol})$ in THF $(25 \mathrm{~mL})$ was added dropwise to the refluxing mixture of $\mathrm{LiAlH}_{4}(52 \mathrm{mmol})$ in THF $(50 \mathrm{~mL})$. The mixture was refluxed for $3 \mathrm{~h}$, and then stirred overnight at rt. The mixture was quenched with saturated $\mathrm{MgSO}_{4}$ solution, and the aluminate salts were filtered. The salts were extracted several times with ether. The combined organic layers were concentrated to afford the crude piperazine as oil. Yield: $86 \%$. $[\alpha]_{\mathrm{D}}=-5.10^{\circ}\left(\mathrm{c}=0.56, \mathrm{CHCl}_{3}\right),\left\{\right.$ lit. $\left.^{1}[\alpha]_{\mathrm{D}}=-5.5^{\circ}\left(\mathrm{C}=0.51, \mathrm{CHCl}_{3}\right)\right\}$ ${ }^{1} \mathrm{H}-\mathrm{NMR}\left(\mathrm{CDCl}_{3}\right): \delta=1.00(\mathrm{~d}, \mathrm{~J}=6.20 \mathrm{~Hz}, 3 \mathrm{H}), 1.66(\mathrm{~m}, 1 \mathrm{H}), 1.95-2.05(\mathrm{~m}, 1 \mathrm{H}), 2.74-$ $2.94(\mathrm{~m}, 5 \mathrm{H}), 3.48(\mathrm{~s}, 2 \mathrm{H}), 7.24-7.33(\mathrm{~m}, 5 \mathrm{H})$.

\section{(S)-1-Benzyl-3-isobutylpiperazine (4b):}




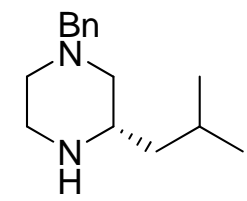

The compound was prepared by the same procedure described above. Yield: $72 \%$, colorless oil, becomes solid on standing. $[\alpha]_{\mathrm{D}}=+3.80^{\circ}\left(\mathrm{C}=2, \mathrm{CHCl}_{3}\right)$,

${ }^{1} \mathrm{H}-\mathrm{NMR}\left(\mathrm{CDCl}_{3}\right): \delta=0.86(\mathrm{~d}, \mathrm{~J}=4.9 \mathrm{~Hz}, 3 \mathrm{H}), 0.88(\mathrm{~d}, \mathrm{~J}=4.9 \mathrm{~Hz}, 3 \mathrm{H}), 1.05-1.28(\mathrm{~m}$, $2 \mathrm{H}), 1.57-1.73(\mathrm{~m}, 2 \mathrm{H}), 1.95-1.97(\mathrm{dt}, \mathrm{J}=4.1 \mathrm{~Hz}, \mathrm{~J}=10.7 \mathrm{~Hz}, 1 \mathrm{H}), 2.70-2.98(\mathrm{~m}, 5 \mathrm{H})$, $3.50(\mathrm{q}, \mathrm{J}=7.9 \mathrm{~Hz}, 2 \mathrm{H}), 7.21-7.33(\mathrm{~m}, 5 \mathrm{H})$.

${ }^{13} \mathrm{C}-\mathrm{NMR}\left(\mathrm{CDCl}_{3}\right): \delta=22.4(+), 23.3(+), 24.3(+), 43.8(-), 45.9(-), 52.8(+), 54.0(-)$, $60.6(-), 63.4(-), 127.0(+), 128.2(+), 129.0(+), 129.3(+), 138.1\left(\mathrm{C}_{\text {quat }}\right)$. MS [CI-MS; $\left.\mathrm{NH}_{3}\right]=233.2\left[\mathrm{M}-\mathrm{H}^{+}\right](100)$.

\section{(S)-1, 3-Dibenzylpiperazine (4c):}

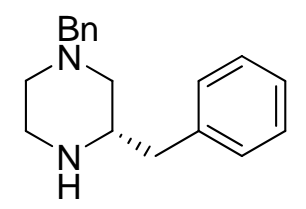

The compound was prepared by the same procedure described above. Yield: $70 \%$, colorless oil, become solid upon standing.

${ }^{1} \mathrm{H}-\mathrm{NMR}\left(\mathrm{CDCl}_{3}\right): \delta=2.89(\mathrm{~d}, \mathrm{~J}=17.6 \mathrm{~Hz}, 1 \mathrm{H}), 3.12(\mathrm{~d}, \mathrm{~J}=4.5 \mathrm{~Hz}, 1 \mathrm{H}), 3.15(\mathrm{~d}, \mathrm{~J}=4.5$ $\mathrm{Hz}, 1 \mathrm{H}), 3.23(\mathrm{~d}, \mathrm{~J}=6.2 \mathrm{~Hz}, 1 \mathrm{H}), 3.25(\mathrm{~d}, \mathrm{~J}=6.2 \mathrm{~Hz}, 1 \mathrm{H}), 3.48(\mathrm{~d}, \mathrm{~J}=17.6 \mathrm{~Hz}, 1 \mathrm{H}), 4.40$ (m, 1H), 4.60 (s, 2H), 7.13-7.39 (m, 10H).

${ }^{13} \mathrm{C}-\mathrm{NMR}\left(\mathrm{CDCl}_{3}\right): \delta=40.9(-), 48.4(-), 49.7(-), 56.5(+), 127.5(+), 128.1(+), 128.7$ $(+), 128.9(+), 130.1(+), 134.8\left(\mathrm{C}_{\text {quat }}\right), 134.9\left(\mathrm{C}_{\text {quat }}\right), 165.3\left(\mathrm{C}_{\text {quat }}\right), 166.3\left(\mathrm{C}_{\text {quat }}\right)$. MS [CI-MS; $\left.\mathrm{NH}_{3}\right]=312.2\left[\mathrm{M}-\mathrm{NH}_{4}{ }^{+}\right](100), 295.1\left[\mathrm{M}-\mathrm{H}^{+}\right](20)$. 


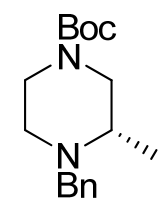

A mixture of compound 4a $(9.51 \mathrm{~g}, 0.05 \mathrm{~mol}), 10 \%$ palladium on carbon $(1.2 \mathrm{~g})$ and $\mathrm{CH}_{3} \mathrm{COOH}(0.5 \mathrm{~mL})$ in $\mathrm{CH}_{3} \mathrm{OH}(100 \mathrm{~mL})$ was stirred under 5 atm of $\mathrm{H}_{2}$ at $\mathrm{rt}$ for $24 \mathrm{~h}$. The catalyst was removed by filtration and washed with $\mathrm{CH}_{3} \mathrm{OH}$. The combined filtrate was concentrated to afford $\mathbf{5 a}$ as colorless solid, with $99 \%$ of yield.

Without any further purification the solid 5a (49.9 mmol) was dissolved in $44 \mathrm{~g} 1$-butanol (0.05 wt. $\% \mathrm{H}_{2} \mathrm{O}$ content), cooled to $0^{\circ} \mathrm{C}$. To this solution $(\mathrm{Boc})_{2} \mathrm{O}(50 \mathrm{mmol})$ in $10 \mathrm{~mL}$ of 1-butanol was added dropwise. The reaction mixture was stirred for $2 \mathrm{~h}$ at $0-5^{\circ} \mathrm{C}$ and $12 \mathrm{~h}$ at $\mathrm{rt}$. 1-Butanol was removed by vacuo to afford crude oil which was dried in high vacuum overnight. The crude product was dissolved in $15 \mathrm{~mL}$ dry $\mathrm{CH}_{3} \mathrm{CN}$. Benzyl bromide (45 mmol) and $\mathrm{K}_{2} \mathrm{CO}_{3}(10 \mathrm{~g})$ were added, and the reaction mixture was stirred at room temperature for $4 \mathrm{~h}$. After consumption of all starting material, the reaction mixture was quenched by $50 \mathrm{~mL}$ of water, and the product was extracted with ethyl acetate $(3 \mathrm{x} 30$ $\mathrm{mL}$ ). The organic phase was dried over $\mathrm{NaSO}_{4}$ and the solvent was removed in vacuo. The product was purified by column chromatography, eluting with petrol ether and ethyl acetate (4:1) to afford $\mathbf{6 a}$ as colorless oil. The yield over two steps was $89 \%$.

${ }^{1} \mathrm{H}-\mathrm{NMR}\left(\mathrm{CDCl}_{3}\right): \delta=1.13(\mathrm{~d}, \mathrm{~J}=6.3 \mathrm{~Hz}, 3 \mathrm{H}), 1.45(\mathrm{~s}, 9 \mathrm{H}), 2.07(\mathrm{~m}, 1 \mathrm{H}), 2.44(\mathrm{~m}, 1 \mathrm{H})$, 2.61-2.67 (m, 1H), 2.78-2.95 (m, 1H), 3.02-3.21 (m, 2H), 3.16-3.80 (m, 2H), 7.23-7.36 $(\mathrm{m}, 5 \mathrm{H})$.

\section{(S)-1-Benzyl-2methylpiperazine (7a)}

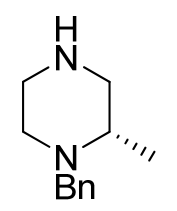

The compound 6a $(1 \mathrm{~g})$ was dissolved in $20 \mathrm{~mL}$ of $\mathrm{CH}_{2} \mathrm{Cl}_{2}$ and $\mathrm{HCl}$ saturated ether (10 $\mathrm{mL}$ ) and the reaction mixture was stirred until all the starting material was consumed (checked by TLC). After completion of the reaction, the solvent was removed in vacuo to 
afford the product as $\mathrm{HCl}$ salt. Then the product was passed through a strongly basic ionexchange column to give the pure product as light yellow oil quantitatively.

\section{General Procedure for Cu (I) mediated C-N Coupling:}

An oven dried Schlenk flask was charged with $\mathrm{CuBr} \bullet \mathrm{Me}_{2} \mathrm{~S}$ (41 mg, $0.2 \mathrm{mmol}$ ) and then it was preheated at $50-60^{\circ} \mathrm{C}$ under high vacuum for the removal of complexed $\mathrm{Me}_{2} \mathrm{~S}$. It was backfilled with nitrogen, then 1,1'-binaphthyl-2,2'-diol (57 mg, $0.2 \mathrm{mmol}$ ), $\mathrm{Cs}_{2} \mathrm{CO}_{3}(424 \mathrm{mg}, 2 \mathrm{mmol})$, and amine $(1.5 \mathrm{mmol})$ were added to the flask. Aryl halide (1 mmol) and DMF ( $1 \mathrm{~mL})$ were added to the flask under nitrogen atmosphere. The mixture was allowed to stir under nitrogen atmosphere at $\mathrm{rt}$ for $24 \mathrm{~h}$. After completion of the reaction, the mixture was diluted with diethyl ether and the solution was filtered to remove insoluble inorganic salts. Removal of the solvent at rotary evaporator, followed by column chromatography on silica gel, using petroleum ether/diethylether (60:1 to 4:1) as eluent afforded the desired product.

\section{(S)-4-Benzyl-1-(4-bromo-3-methylphenyl)-2-methylpiperazine (9a):}

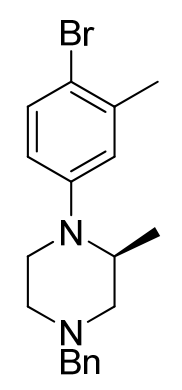

${ }^{1} \mathrm{H}-\mathrm{NMR}\left(\mathrm{CDCl}_{3}\right) \delta: 1.08(\mathrm{~d}, \mathrm{~J}=6.6 \mathrm{~Hz}, 3 \mathrm{H}), 2.33(\mathrm{~s}, 3 \mathrm{H}), 2.31-2.37$ (dd, J = 3.8, J = 3.9 $\mathrm{Hz}, 1 \mathrm{H}), 2.45-2.48(\mathrm{dd}, \mathrm{J}=3.8, \mathrm{~J}=3.9 \mathrm{~Hz}, 1 \mathrm{H}), 2.60-2.63(\mathrm{dd}, \mathrm{J}=1.7, \mathrm{~J}=1.7 \mathrm{~Hz}, 1 \mathrm{H})$, 2.81-2.83 (m, 1H), 3.10-3.21 (m, 2H), $3.48(\mathrm{~d}, \mathrm{~J}=13.2 \mathrm{~Hz}, 1 \mathrm{H}), 3.59$ (d, J = 13.2 Hz, $1 \mathrm{H}), 3.75-3.86(\mathrm{~m}, 1 \mathrm{H}), 6.60-6.62(\mathrm{dd}, \mathrm{J}=3.2, \mathrm{~J}=3.2 \mathrm{~Hz}, 1 \mathrm{H}), 6.77(\mathrm{~d}, \mathrm{~J}=3.2 \mathrm{~Hz}, 1 \mathrm{H})$, 7.24-7.42 (m, 6H).

${ }^{13} \mathrm{C}-\mathrm{NMR}\left(\mathrm{CDCl}_{3}\right) \delta: 13.2(+), 23.3(+), 44.6(-), 51.2(+), 53.4(-), 58.6(-), 62.8(-)$, $114.1(+), 116.0(+), 119.3(+), 127.0(+), 128.2(+), 128.8(+), 132.5(+), 138.1(+)$, 
$138.5(+), 149.6\left(\mathrm{C}_{\text {quat }}\right)$ - $\mathrm{MS}$ [CI-MS; $\left.\left(\mathrm{NH}_{3}\right)\right]=359.1,361.1\left[\mathrm{M}-\mathrm{H}^{+}\right](100), 281.3[\mathrm{M}-$ $\left.\mathrm{Br}^{+}\right](15)$.

(S)-4-Benzyl-1-(4-bromo-3-methylphenyl)-2-isobutylpiperazine (9b):

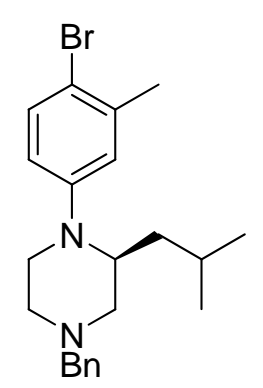

${ }^{1} \mathrm{H}-\mathrm{NMR}\left(\mathrm{CDCl}_{3}\right) \delta: 0.81(\mathrm{~d}, \mathrm{~J}=6.5 \mathrm{~Hz}, 3 \mathrm{H}), 0.83(\mathrm{~d}, \mathrm{~J}=6.5 \mathrm{~Hz}, 3 \mathrm{H}), 1.04-1.08(\mathrm{~m}$, 1H), 1.31-1.37 (m, 1H), 1.90-1.94 (m, 1H), 2.24-2.32 (m, 2H), 2.33 (s, 3H), 2.75-2.78 $(\mathrm{dt}, \mathrm{J}=11.1, \mathrm{~J}=2.1 \mathrm{~Hz}, 1 \mathrm{H}), 2.87-2.88(\mathrm{dq}, \mathrm{J}=11.1, \mathrm{~J}=2.1 \mathrm{~Hz}, 1 \mathrm{H}), 3.12-3.14(\mathrm{dt}, \mathrm{J}=$ 11.6, $\mathrm{J}=3.2 \mathrm{~Hz}, 1 \mathrm{H}), 3.22-3.24(\mathrm{td}, \mathrm{J}=3.2, \mathrm{~J}=11.6 \mathrm{~Hz}, 1 \mathrm{H}), 3.41(\mathrm{~d}, \mathrm{~J}=13.3 \mathrm{~Hz}, 1 \mathrm{H})$, $3.63(\mathrm{~d}, \mathrm{~J}=13.3 \mathrm{~Hz}, 1 \mathrm{H}), 3.70-3.74(\mathrm{~m}, 1 \mathrm{H}), 6.56(\mathrm{dd}, \mathrm{J}=2.8, \mathrm{~J}=2.6 \mathrm{~Hz}, 1 \mathrm{H}), 6.70(\mathrm{~d}, \mathrm{~J}$ $=2.6 \mathrm{~Hz}, 1 \mathrm{H}), 7.24-7.37(\mathrm{~m}, 6 \mathrm{H})$.

${ }^{13} \mathrm{C}-\mathrm{NMR}\left(\mathrm{CDCl}_{3}\right)$ ): $21.5(+), 23.6(+), 23.8(+), 25.1(+), 35.2(-), 43.4(-), 53.3(+)$, $53.8(-), 54.8(-), 62.8(-), 112.9(+), 114.8(+), 117.9(+), 127.0(+), 128.2(+), 128.8(+)$, $132.5(+), 138.0(+), 138.6(+), 149.2\left(\mathrm{C}_{\text {quat }}\right)$ - - MS [CI-MS; $\left.\left(\mathrm{NH}_{3}\right)\right]=401.1,403.1[\mathrm{M}-$ $\left.\mathrm{H}^{+}\right](100), 323.2\left[\mathrm{M}-\mathrm{Br}^{+}\right](15)$.

(S)-2, 4-Dibenzyl-1-(4-bromo-3-methylphenyl) piperazine (9c):

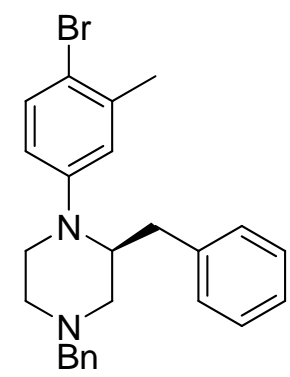


${ }^{1} \mathrm{H}-\mathrm{NMR}\left(\mathrm{CDCl}_{3}\right) \delta: 2.13(\mathrm{dd}, \mathrm{J}=11.2, \mathrm{~J}=3.0 \mathrm{~Hz}, 1 \mathrm{H}), 2.30(\mathrm{dt}, \mathrm{J}=11.2, \mathrm{~J}=3.5 \mathrm{~Hz}$, $1 \mathrm{H}), 2.39$ (s, 3H), $2.50(\mathrm{dd}, \mathrm{J}=12.8, \mathrm{~J}=2.3 \mathrm{~Hz}, 1 \mathrm{H}), 2.87-2.88(\mathrm{dq}, \mathrm{J}=11.1, \mathrm{~J}=2.1 \mathrm{~Hz}$, $1 \mathrm{H}), 3.12-3.14(\mathrm{dt}, \mathrm{J}=11.6, \mathrm{~J}=3.2 \mathrm{~Hz}, 1 \mathrm{H}), 3.22-3.24(\mathrm{td}, \mathrm{J}=3.2, \mathrm{~J}=11.6 \mathrm{~Hz}, 1 \mathrm{H}), 3.41$ $(\mathrm{d}, \mathrm{J}=13.3 \mathrm{~Hz}, 1 \mathrm{H}), 3.63(\mathrm{~d}, \mathrm{~J}=13.3 \mathrm{~Hz}, 1 \mathrm{H}), 3.70-3.74(\mathrm{~m}, 1 \mathrm{H}), 6.56(\mathrm{dd}, \mathrm{J}=2.8, \mathrm{~J}=$ $2.6 \mathrm{~Hz}, 1 \mathrm{H}), 6.70(\mathrm{~d}, \mathrm{~J}=2.6 \mathrm{~Hz}, 1 \mathrm{H}), 7.24-7.37(\mathrm{~m}, 6 \mathrm{H})$.

${ }^{13} \mathrm{C}-\mathrm{NMR}\left(\mathrm{CDCl}_{3}\right)$ ): $21.5(+), 23.6(+), 23.8(+), 25.1(+), 35.2(-), 43.4(-), 53.3(+)$, $53.8(-), 54.8(-), 62.8(-), 112.9(+), 114.8(+), 117.9(+), 127.0(+), 128.2(+), 128.8(+)$, $132.5(+), 138.0(+), 138.6(+), 149.2\left(\mathrm{C}_{\text {quat }}\right)$ - - MS [CI-MS; $\left.\left(\mathrm{NH}_{3}\right)\right]=401.1,403.1$ [M$\left.\mathrm{H}^{+}\right](100), 323.2\left[\mathrm{M}-\mathrm{Br}^{+}\right](15)$.

\section{General Procedure for Pd (0) mediated C-N Coupling:}

An ovendried Schlenk flask was charged with the aryl iodide $(0.5 \mathrm{mmol})$, amine $(0.6$ mmol), NaO- ${ }^{t} \mathrm{Bu}$ (67 mg, $0.7 \mathrm{mmol}$ ), $\mathrm{Pd}_{2}(\mathrm{dba})_{3} \cdot \mathrm{CHCl}_{3}(2.3 \mathrm{mg}, 0.0025 \mathrm{mmol}, 1 \mathrm{~mol} \%$ of Pd), BINAP (4.7 mg, $0.0075 \mathrm{mmol})$ and purged with argon. Toluene $(1 \mathrm{~mL})$ was added, and the reaction mixture was heated at $120-125^{\circ} \mathrm{C}$ under argon for $8 \mathrm{~h}$ until the reaction had proceeded to completion as judged by GC or TLC analysis. The reaction mixture was taken up in ethyl acetate $(20 \mathrm{~mL})$, filtered, and concentrated in vacuo. The crude product was then purified by flash chromatography on silica gel.

(S)-1-Benzyl-4-(4-((S)-4-benzyl-2-methylpiperazine-1-yl)-2-methylphenyl)-2methylpiperazine (10a)

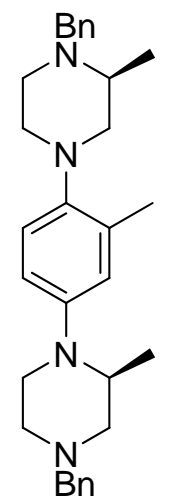


${ }^{1} \mathrm{H}-\mathrm{NMR}\left(\mathrm{CDCl}_{3}\right) \delta: 1.05(\mathrm{~d}, \mathrm{~J}=6.4 \mathrm{~Hz}, 3 \mathrm{H}), 1.24(\mathrm{~d}, \mathrm{~J}=5.7 \mathrm{~Hz}, 3 \mathrm{H}), 2.30(\mathrm{~s}, 3 \mathrm{H}), 2.34-$ $2.40(\mathrm{~m}, 1 \mathrm{H}), 2.45-2.51(\mathrm{~m}, 2 \mathrm{H}), 2.57-2.61(\mathrm{~m}, 1 \mathrm{H}), 2.63-2.76(\mathrm{~m}, 3 \mathrm{H}), 2.78-2.84(\mathrm{~m}$, 2H), 2.87-2.91 (m, 1H), 2.95-2.98 (m, 1H), 3.11-3.14 (m, 2H), $3.29(\mathrm{~d}, \mathrm{~J}=13.0 \mathrm{~Hz}, 1 \mathrm{H})$, $3.51(\mathrm{~d}, \mathrm{~J}=13.0 \mathrm{~Hz}, 1 \mathrm{H}), 3.61(\mathrm{~d}, \mathrm{~J}=13.0 \mathrm{~Hz}, 1 \mathrm{H}), 3.63-3.67(\mathrm{~m}, 1 \mathrm{H}), 4.11(\mathrm{~d}, \mathrm{~J}=13.0$ $\mathrm{Hz}, 1 \mathrm{H}), 6.77-6.79(\mathrm{dd}, \mathrm{J}=8.5 \mathrm{~Hz}, \mathrm{~J}=2.6 \mathrm{~Hz}, 1 \mathrm{H}), 6.83(\mathrm{~d}, \mathrm{~J}=2.6 \mathrm{~Hz}, 1 \mathrm{H}), 6.97$ (d, J = $8.5 \mathrm{~Hz}, 1 \mathrm{H}), 7.26-7.41(\mathrm{~m}, 10 \mathrm{H})$.

${ }^{13} \mathrm{C}-\mathrm{NMR}\left(\mathrm{CDCl}_{3}\right)$ 8: $14.1(+), 18.0(+), 47.2(-), 51.5(-), 52.0(+), 52.4(-), 53.7(-), 55.7$ $(+), 58.2(-), 59.3(-), 59.6(-), 62.9(-), 116.6(+), 119.6(+), 121.5(+), 126.8(+), 126.9$ $(+), 128.2(+), 128.9(+), 129.1(+), 133.4(+), 138.5\left(\mathrm{C}_{\text {quat }}\right), 139.0\left(\mathrm{C}_{\text {quat }}\right), 145.0\left(\mathrm{C}_{\text {quat }}\right)$, $146.2\left(\mathrm{C}_{\text {quat }}\right) .-\mathrm{MS}\left[\mathrm{CI}-\mathrm{MS} ;\left(\mathrm{NH}_{3}\right)\right]=469.2\left[\mathrm{M}-\mathrm{H}^{+}\right](100)$.

(S)-1-Benzyl-4-(4-((S)-4-benzyl-2-isobutylpiperazine-1-yl)-2-methylphenyl)-2isobutylpiperazine (10b)

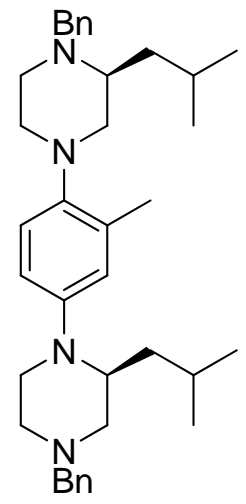

${ }^{1} \mathrm{H}-\mathrm{NMR}\left(\mathrm{CDCl}_{3}\right) \delta: 0.80-0.84(\mathrm{dd}, \mathrm{J}=6.7, \mathrm{~J}=13.5 \mathrm{~Hz}, 6 \mathrm{H}), 0.89-0.96(\mathrm{dd}, \mathrm{J}=6.7, \mathrm{~J}=$ $21.0 \mathrm{~Hz}, 6 \mathrm{H}), 1.07-1.13(\mathrm{~m}, 1 \mathrm{H}), 1.33-1.40(\mathrm{~m}, 1 \mathrm{H}), 1.57-1.67(\mathrm{~m}, 2 \mathrm{H}), 1.81-1.87(\mathrm{~m}$, $1 \mathrm{H}), 2.26(\mathrm{~s}, 3 \mathrm{H}), 2.32-2.41(\mathrm{~m}, 3 \mathrm{H}), 2.58-2.65(\mathrm{~m}, 2 \mathrm{H}), 2.67-2.74(\mathrm{~m}, 1 \mathrm{H}), 2.75-2.86$ (m, 4H), $3.03(\mathrm{~d}, \mathrm{~J}=9.1 \mathrm{~Hz}, 1 \mathrm{H}), 3.10-3.17(\mathrm{~m}, 1 \mathrm{H}), 3.18-3.22(\mathrm{~m}, 1 \mathrm{H}), 3.28(\mathrm{~d}, \mathrm{~J}=12.5$ $\mathrm{Hz}, 1 \mathrm{H}), 3.42(\mathrm{~d}, \mathrm{~J}=12.5 \mathrm{~Hz}, 1 \mathrm{H}), 3.61-3.67(\mathrm{~m}, 3 \mathrm{H}), 4.07(\mathrm{~d}, \mathrm{~J}=13.0 \mathrm{~Hz}, 1 \mathrm{H}), 6.65-$ $6.69(\mathrm{dd}, \mathrm{J}=8.6 \mathrm{~Hz}, \mathrm{~J}=2.6 \mathrm{~Hz}, 1 \mathrm{H}), 6.7(\mathrm{~d}, \mathrm{~J}=2.6 \mathrm{~Hz}, 1 \mathrm{H}), 6.9(\mathrm{~d}, \mathrm{~J}=8.6 \mathrm{~Hz}, 1 \mathrm{H})$, 7.23-7.40 (m, 10H).

${ }^{13} \mathrm{C}-\mathrm{NMR}\left(\mathrm{CDCl}_{3}\right)$ 8: $18.1(+), 21.5(+), 22.2(+), 23.8(+), 23.9(+), 25.1(+), 25.9(+)$, $35.3(-), 38.3(-), 44.8(-), 51.0(-), 51.9(-), 53.6(+), 54.1(-), 55.4(-), 57.2(+), 58.0(-)$, 
$58.9(-), 114.4(+), 119.2(+), 119.8(+), 126.7(+), 126.9(+), 128.1(+), 128.2(+), 128.8$ $(+), 129.0(+), 133.4(+), 133.5\left(\mathrm{C}_{\text {quat }}\right), 138.6(+), 139.4\left(\mathrm{C}_{\text {quat }}\right), 143.9\left(\mathrm{C}_{\text {quat }}\right), 145.9$ $\left(\mathrm{C}_{\text {quat }}\right) .-[\alpha]_{\mathrm{D}}=+140.0^{\circ}(\mathrm{C}=0.50, \mathrm{MeOH}),+132.0^{\circ}\left(\mathrm{C}=0.50, \mathrm{CHCl}_{3}\right)$. - HRMS (EIMS) calculated for $\mathrm{C}_{37} \mathrm{H}_{52} \mathrm{~N}_{4}\left[\mathrm{M}^{+}\right]$: 552.4192; found 552.4182 .

(S)-1, 2-Dibenzyl-4-(4-((S)-4-benzyl-2-isobutylpiperazine-1-yl)-2-methylphenyl) piperazine (10c)

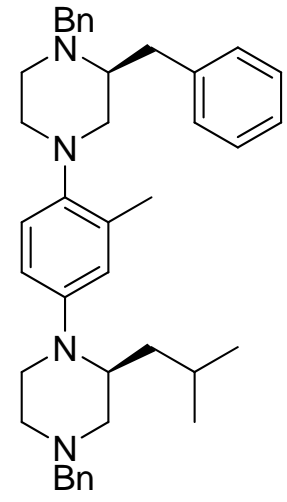

${ }^{1} \mathrm{H}-\mathrm{NMR}\left(\mathrm{CDCl}_{3}\right) \delta:$ 0.92-0.96 (dd, $\left.\mathrm{J}=22.1, \mathrm{~J}=6.1 \mathrm{~Hz}, 6 \mathrm{H}\right), 1.12-1.19(\mathrm{~m}, 1 \mathrm{H}), 1.52-$ $1.73(\mathrm{~m}, 3 \mathrm{H}), 2.17-2.19(\mathrm{dd}, \mathrm{J}=11.1, \mathrm{~J}=2.8 \mathrm{~Hz}, 1 \mathrm{H}), 2.31(\mathrm{~m}, 4 \mathrm{H}), 2.38-2.43(\mathrm{~m}, 1 \mathrm{H})$, 2.63-2.67 (m, 2H), 2.71-2.73 (dt, J = 11.1, J = 1.3 Hz, 1H), 2.81-2.89 (d, J = 11.1 Hz, 1H), $2.96(\mathrm{~d}, \mathrm{~J}=8.5 \mathrm{~Hz}, 1 \mathrm{H}), 3.19(\mathrm{t}, \mathrm{J}=11.8 \mathrm{~Hz}, 1 \mathrm{H}), 3.19-3.32(\mathrm{~m}, 3 \mathrm{H}), 3.40(\mathrm{~d}, \mathrm{~J}=$ $14.0 \mathrm{~Hz}, 1 \mathrm{H}), 3.59$ (d, J = 14.0 Hz, 1H), 3.78-3.81 (dd, J = 10.7, J = 2.4 Hz, 1H), 4.09 (d, $\mathrm{J}=11.8 \mathrm{~Hz}, 1 \mathrm{H}), 6.77-6.80(\mathrm{dd}, \mathrm{J}=2.6, \mathrm{~J}=8.5 \mathrm{~Hz}, 1 \mathrm{H}), 6.82(\mathrm{~d}, \mathrm{~J}=2.4 \mathrm{~Hz}, 1 \mathrm{H}), 6.95-$ $6.97(\mathrm{~m}, 2 \mathrm{H}), 7.03(\mathrm{~d}, \mathrm{~J}=8.5 \mathrm{~Hz}, 1 \mathrm{H}), 7.10-7.25(\mathrm{~m}, 3 \mathrm{H}), 7.25-7.42$ (m, 10H).

${ }^{13} \mathrm{C}-\mathrm{NMR}\left(\mathrm{CDCl}_{3}\right)$ 8: $18.2(+), 22.2(+), 23.9(+), 25.8(+), 32.3(-), 38.5(-), 43.9(-), 51.2$ $(-), 51.9(+), 53.5(-), 53.8(-), 57.26(+), 58.0(-), 58.8(-), 63.0(-), 113.3(+), 114.1(+)$, $117.1\left(^{+}\right), 118.8(+), 120.1(+), 125.7(+), 126.8(+), 127.1\left(^{+}\right), 128.2(+), 129.0(+)$, $129.3(+), 129.4(+), 133.8(+), 138.5\left(\mathrm{C}_{\text {quat }}\right), 139.5\left(\mathrm{C}_{\text {quat }}\right), 140.3\left(\mathrm{C}_{\text {quat }}\right), 144.0\left(\mathrm{C}_{\text {quat }}\right)$, $145.6\left(\mathrm{C}_{\text {quat }}\right) .-\mathrm{MS}\left[\mathrm{CI}-\mathrm{MS} ;\left(\mathrm{NH}_{3}\right)\right]=587.2\left[\mathrm{M}-\mathrm{H}^{+}\right](100)$.

\section{General procedure for benzyl deprotection reaction:}

A mixture of dibenzyl protected compound $(0.05 \mathrm{mmol}), 10 \%$ palladium on carbon (20 mol\%) and $\mathrm{CH}_{3} \mathrm{COOH}$ (2 drops) in $\mathrm{CH}_{3} \mathrm{OH}(3 \mathrm{~mL})$ was stirred under 15 atm of $\mathrm{H}_{2}$ at $\mathrm{rt}$ 
for $24 \mathrm{~h}$. After completion of the reaction the solution was passed over celite to remove the catalyst and washed several times with $\mathrm{CH}_{3} \mathrm{OH}$. The combined filtrate was concentrated to give a hygroscopic solid in quantitative yield

(S)-2-Isobutyl-1-(4-((S)-3-isobutylpiperazine-1-yl)-3-methylphenyl) piperazine (11b)

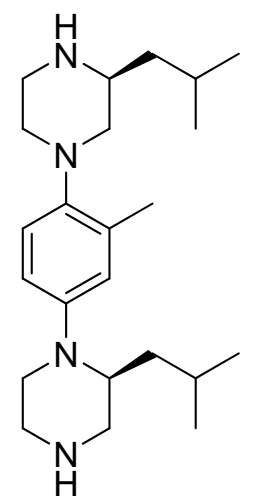

${ }^{1} \mathrm{HNMR}\left(\mathrm{D}_{2} \mathrm{O}\right) \delta: 0.66-0.68(\mathrm{dd}, \mathrm{J}=2.7, \mathrm{~J}=6.3 \mathrm{~Hz}, 6 \mathrm{H}), 0.81-0.84(\mathrm{dd}, \mathrm{J}=2.1, \mathrm{~J}=6.3$ $\mathrm{Hz}, 6 \mathrm{H}), 1.02-1.14(\mathrm{~m}, 1 \mathrm{H}), 1.27-1.62(\mathrm{~m}, 5 \mathrm{H}), 2.19$ (s, 3H), 2.23-2.71 (m, 1H), 2.28$2.94(\mathrm{~m}, 1 \mathrm{H}), 3.10-3.49(\mathrm{~m}, 10 \mathrm{H}), 3.68(\mathrm{~m}, 1 \mathrm{H}), 6.85-7.00(\mathrm{~m}, 3 \mathrm{H})$.

\section{Circular dichroism measurements.}

Measurements were performed on a Jasco J-710 spectrometer. The length of the cylindrical cuvettes was $1 \mathrm{~cm}$. The resolution was $0.2 \mathrm{~nm}$, the band width $1.0 \mathrm{~nm}$, the sensitivity $20 \mathrm{mdeg}$, the response $0.25 \mathrm{~s}$, and the speed $20 \mathrm{~nm} / \mathrm{min}$. Spectra were corrected by the background. The absorption values were measured as molar ellipticity per residue $\left(\operatorname{deg~} \mathrm{cm}^{2} \mathrm{dmol}^{-1}\right)$. 


\section{S-14}

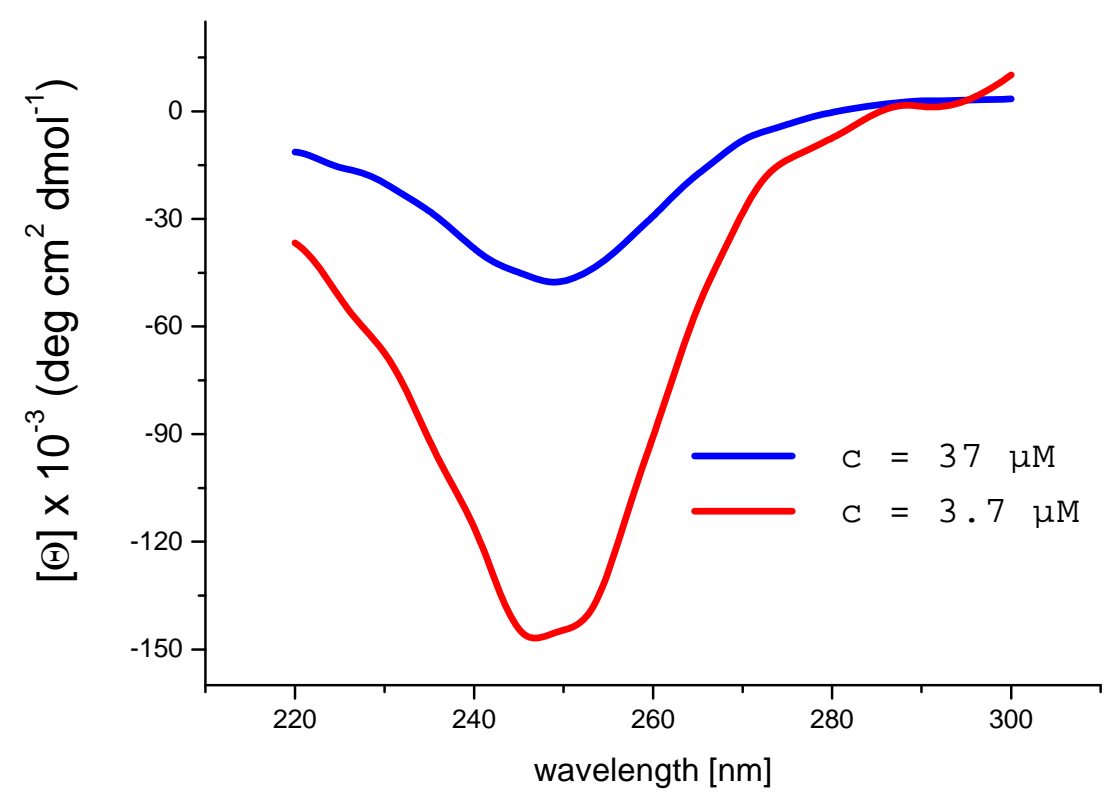

\section{Concentration dependent NMR study}
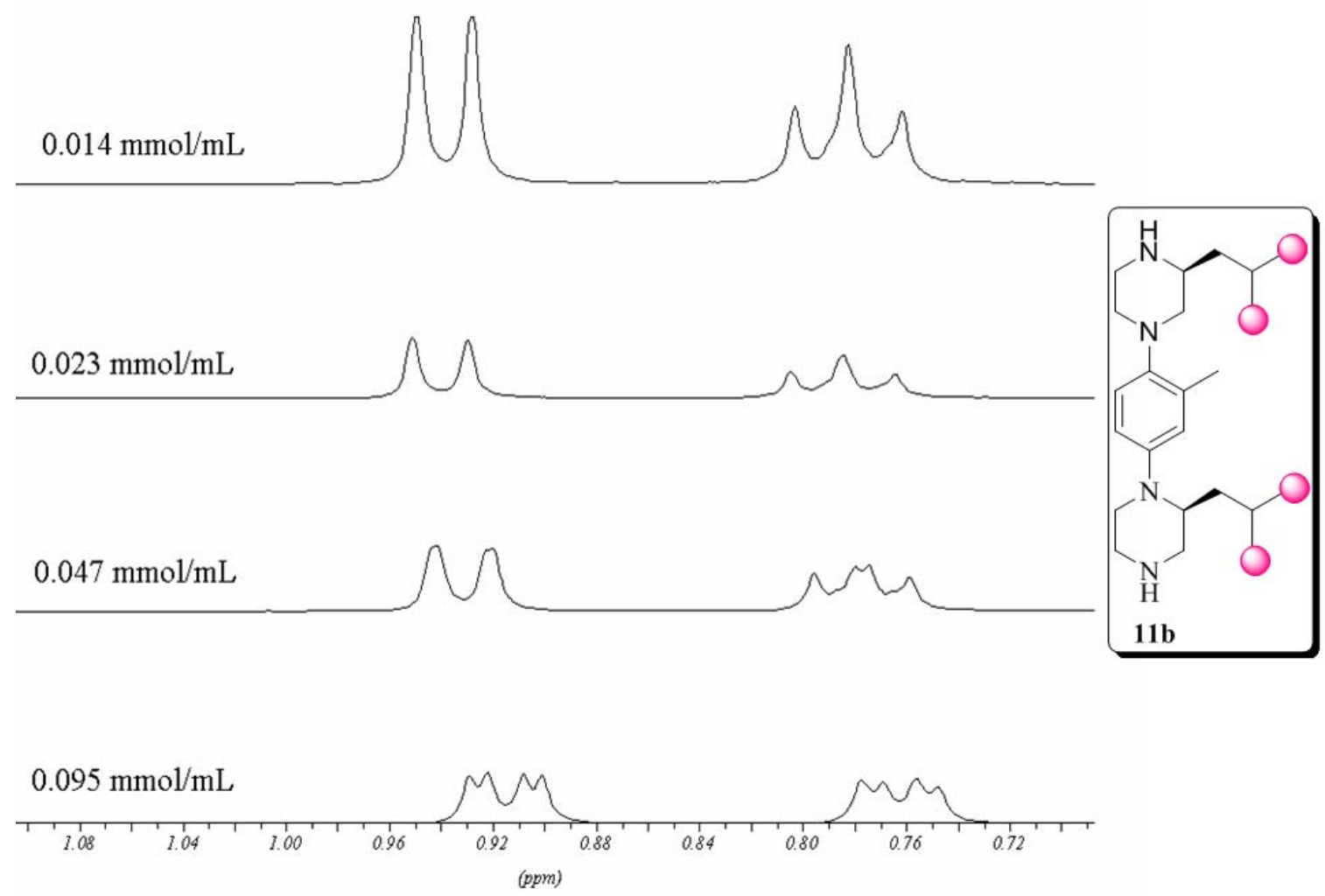
S-15

X-ray diffraction structure of compound 9a

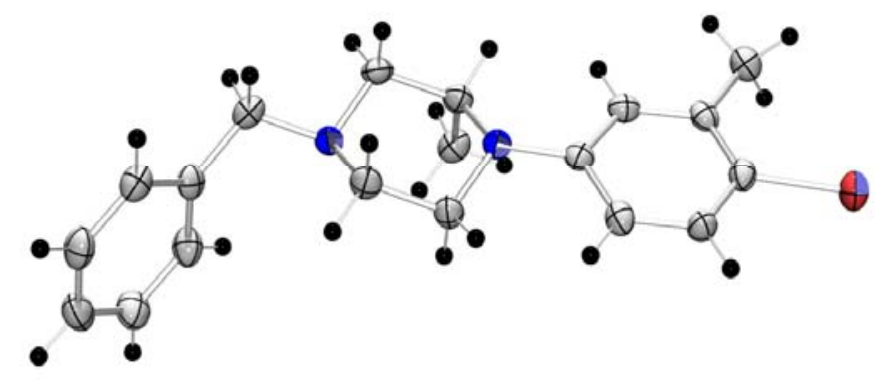

${ }^{1}$ Liu, B.; Xu, G. -Y.; Yang, C. -H.; Wu, X. -H.; Xie, Y. -Y. Syn. Comn. 2004, 34, 4111-4118. 


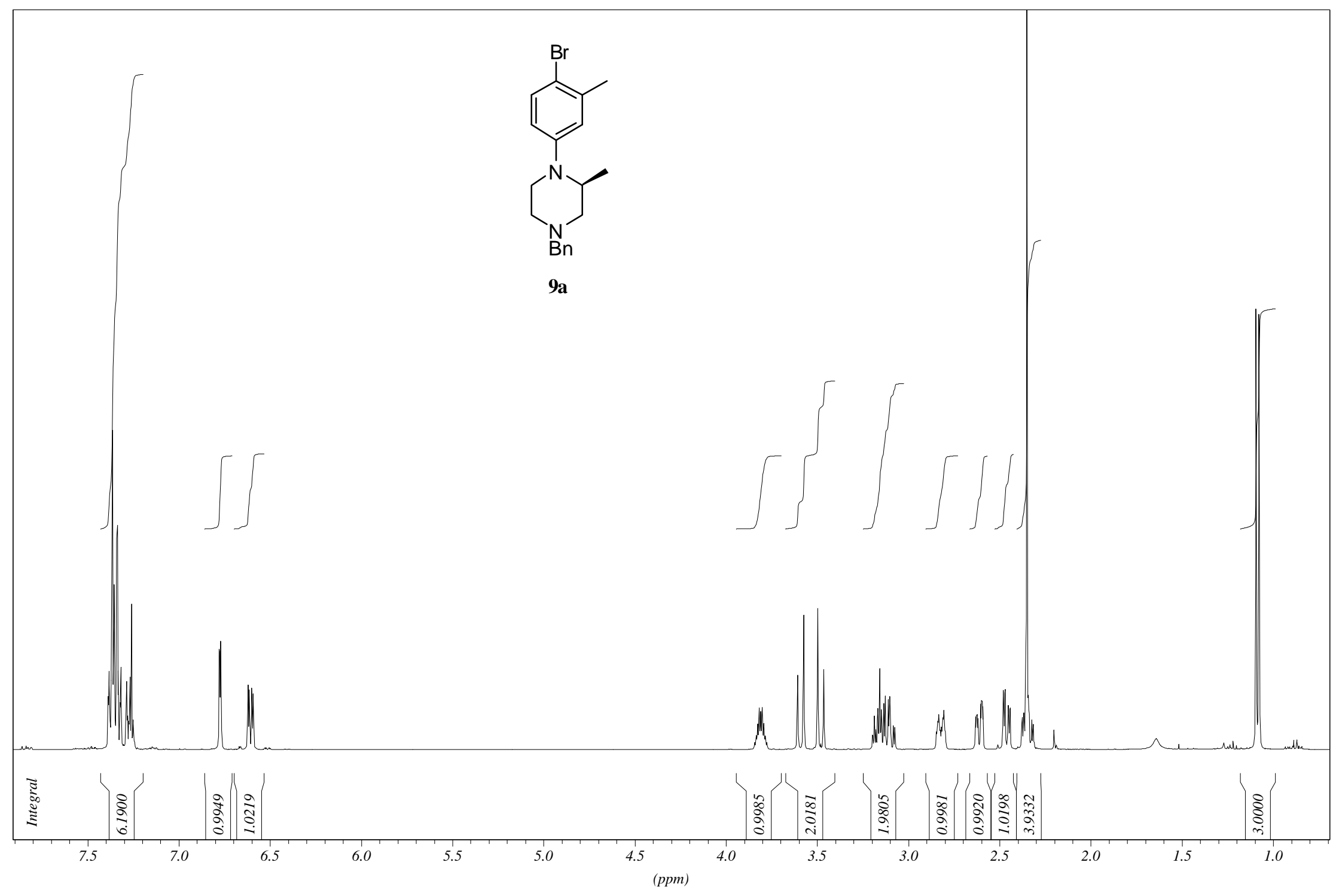




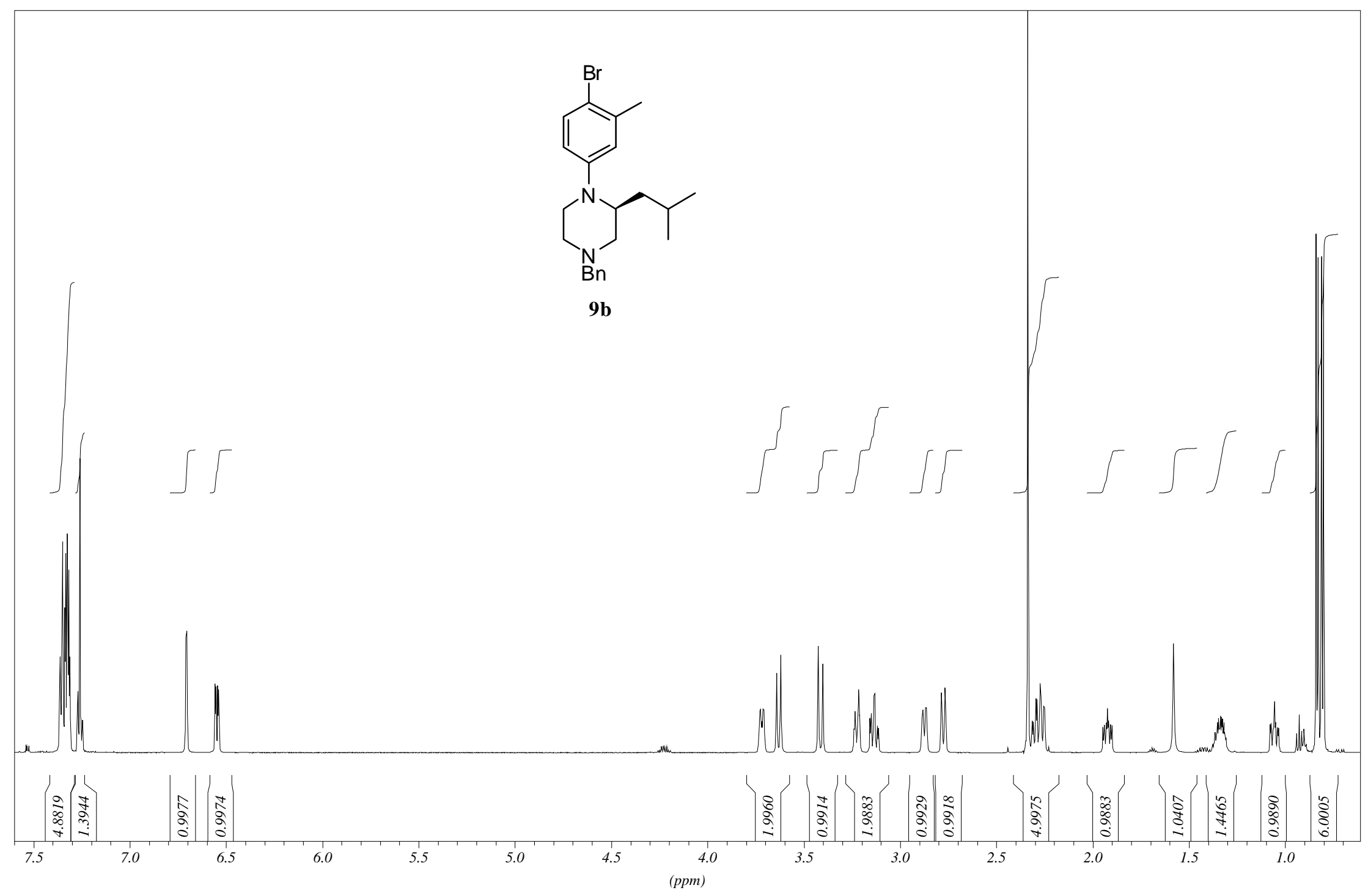




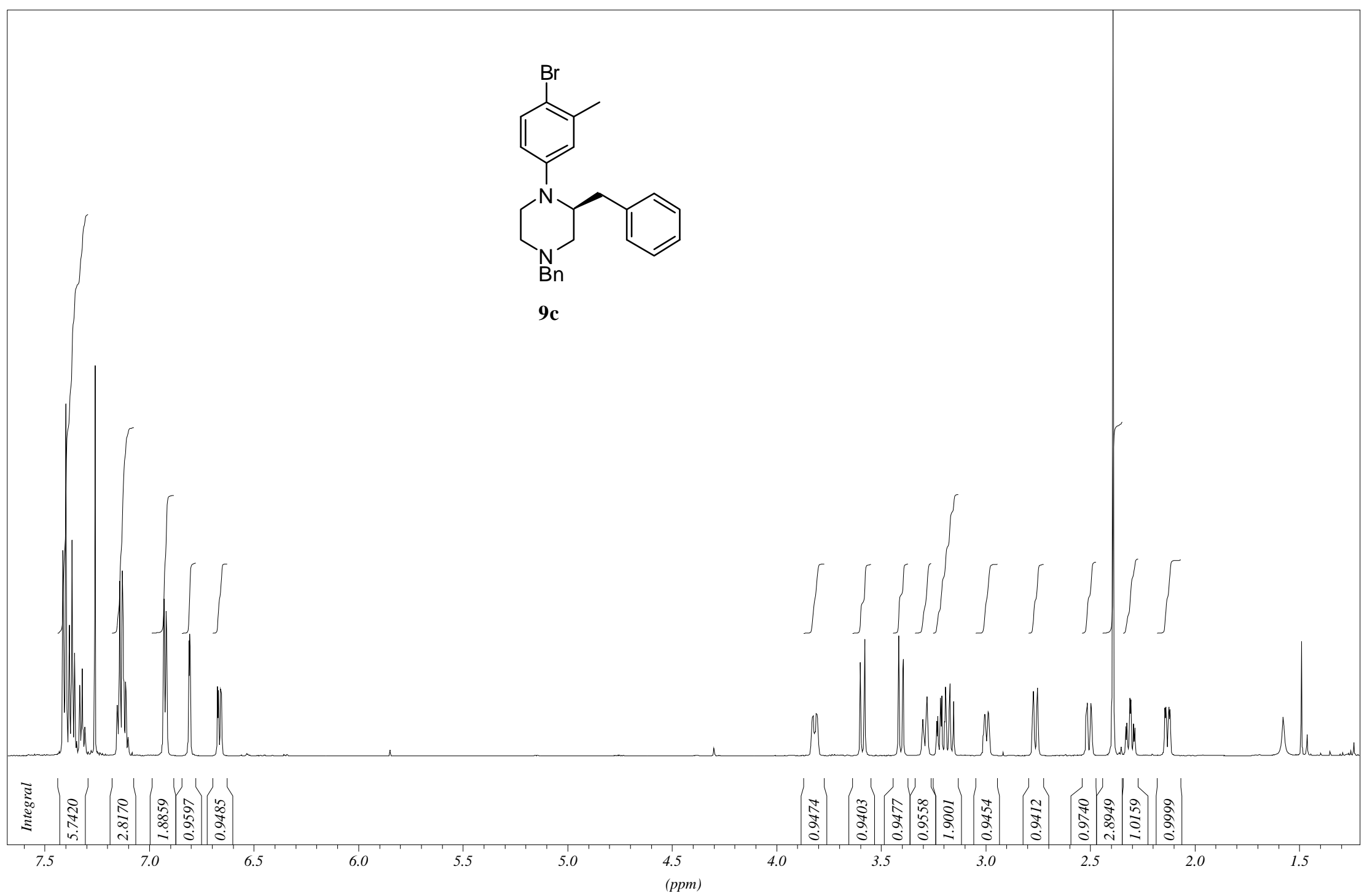




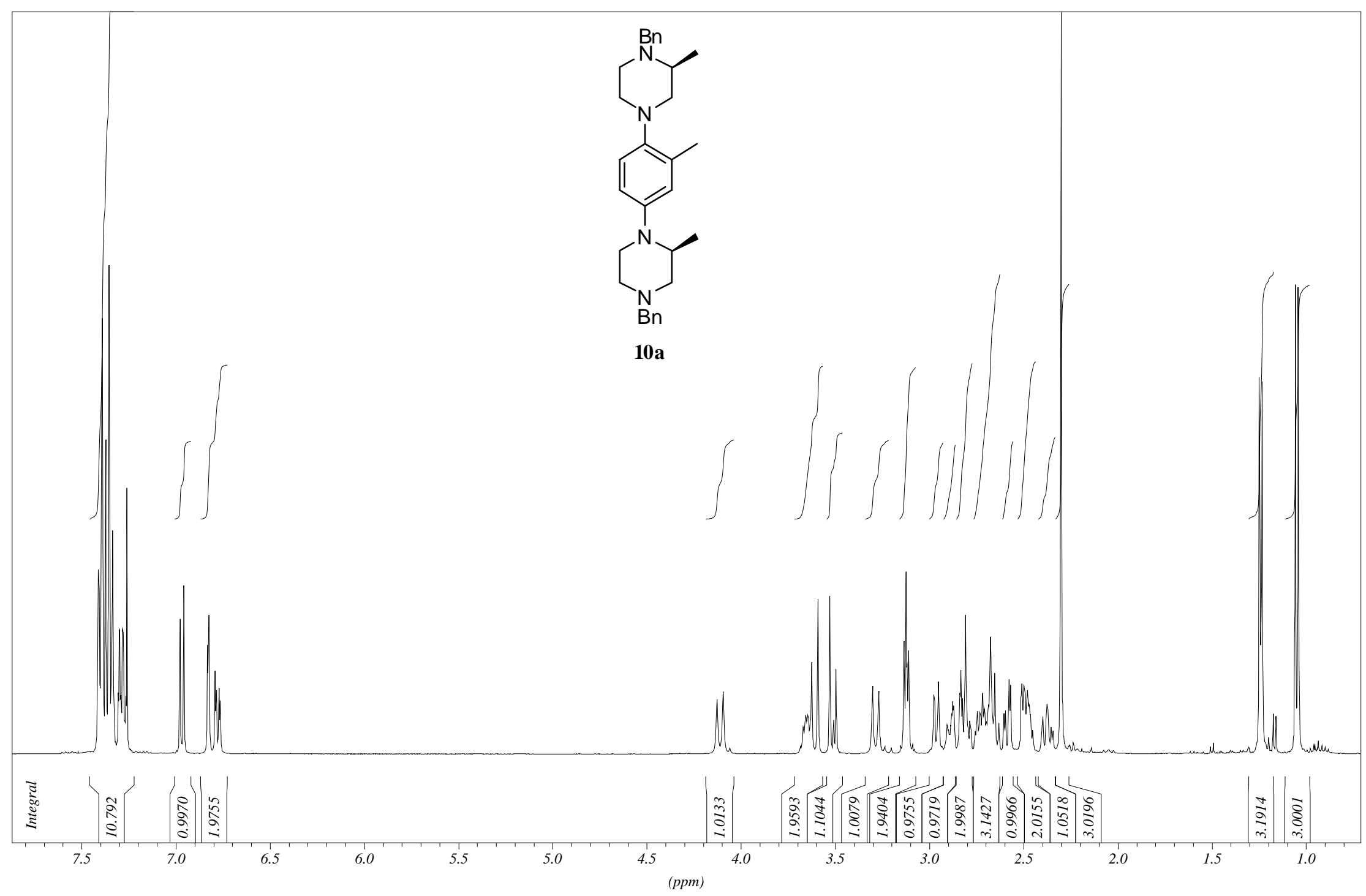




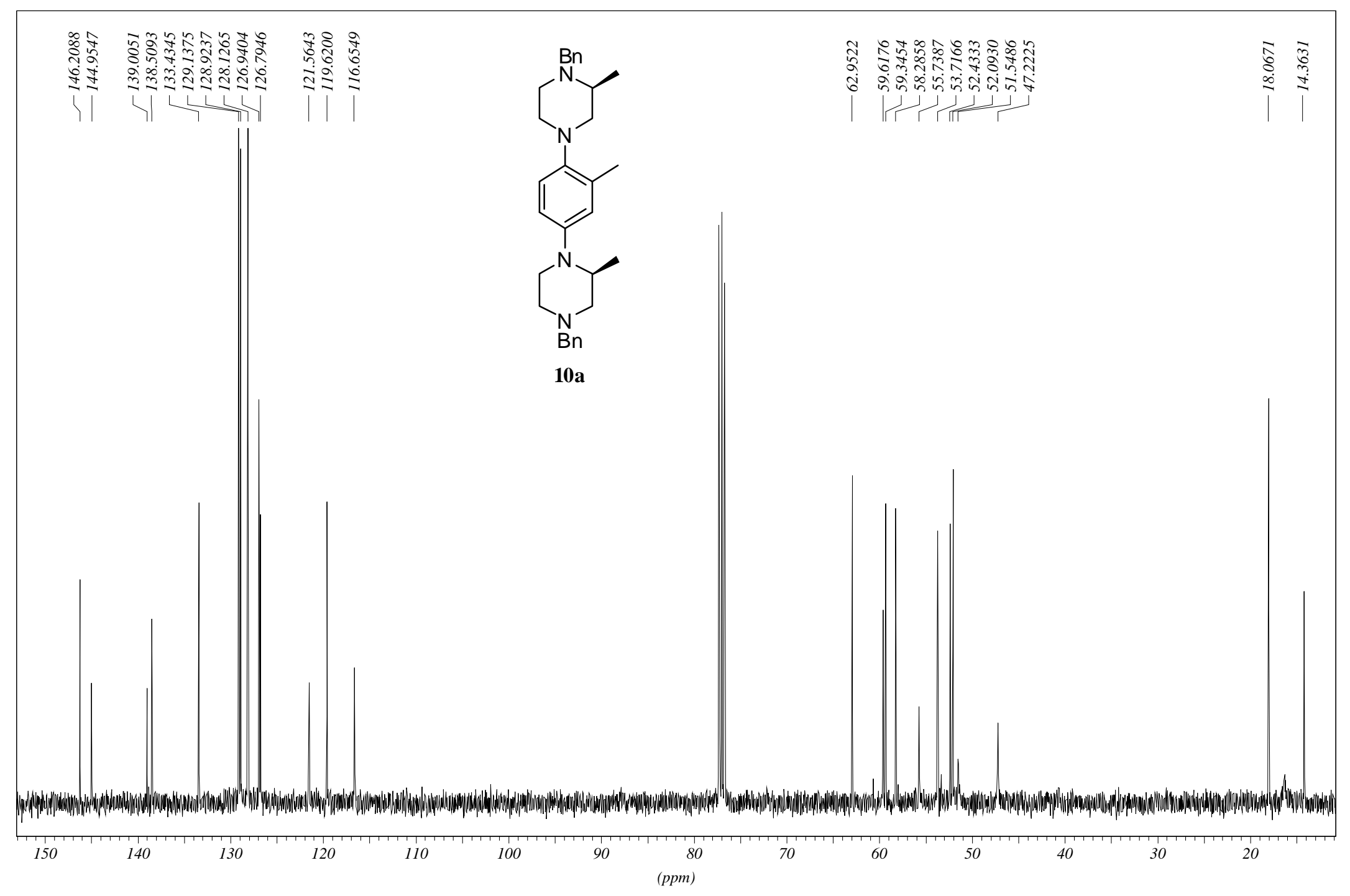




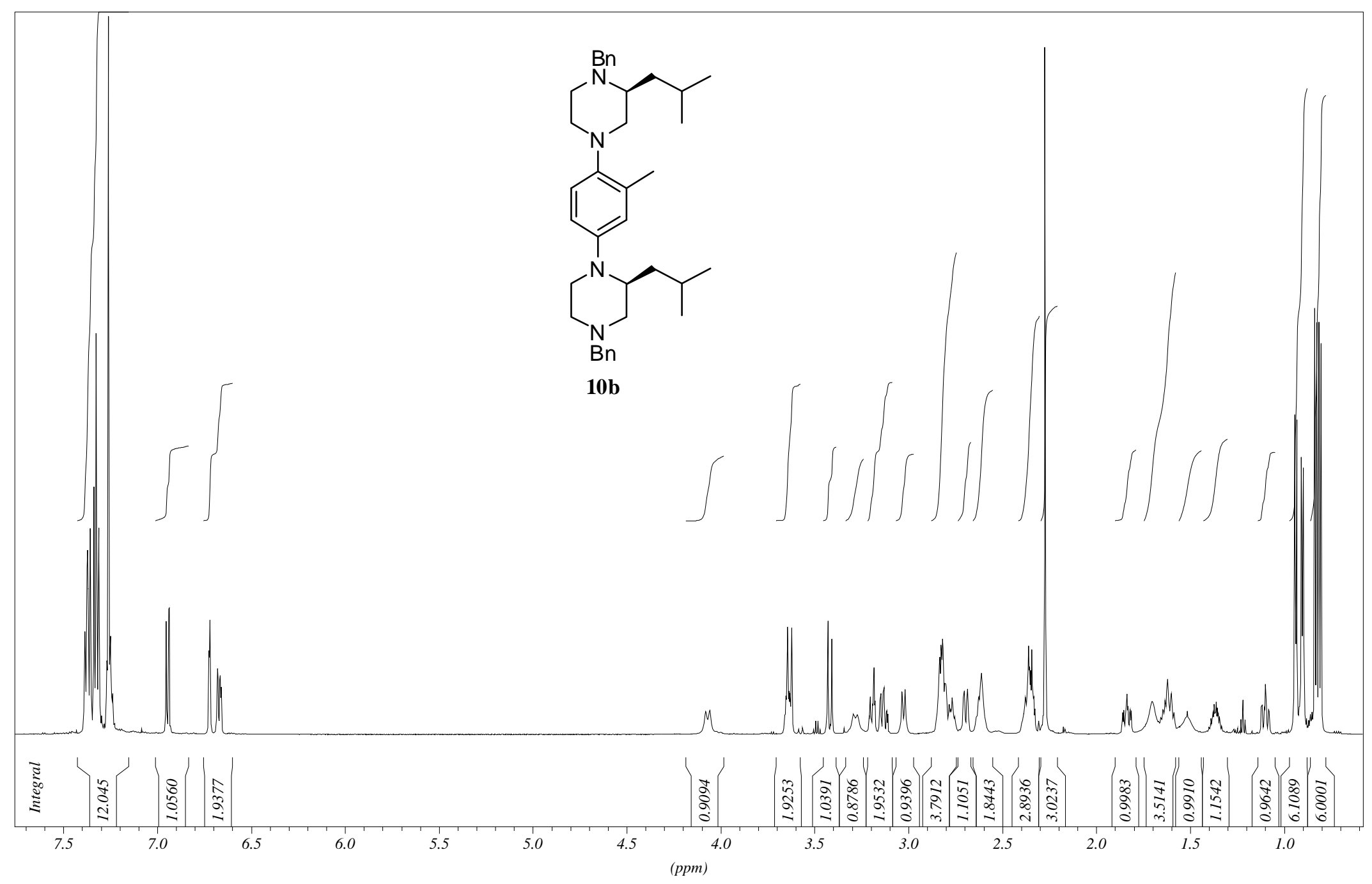




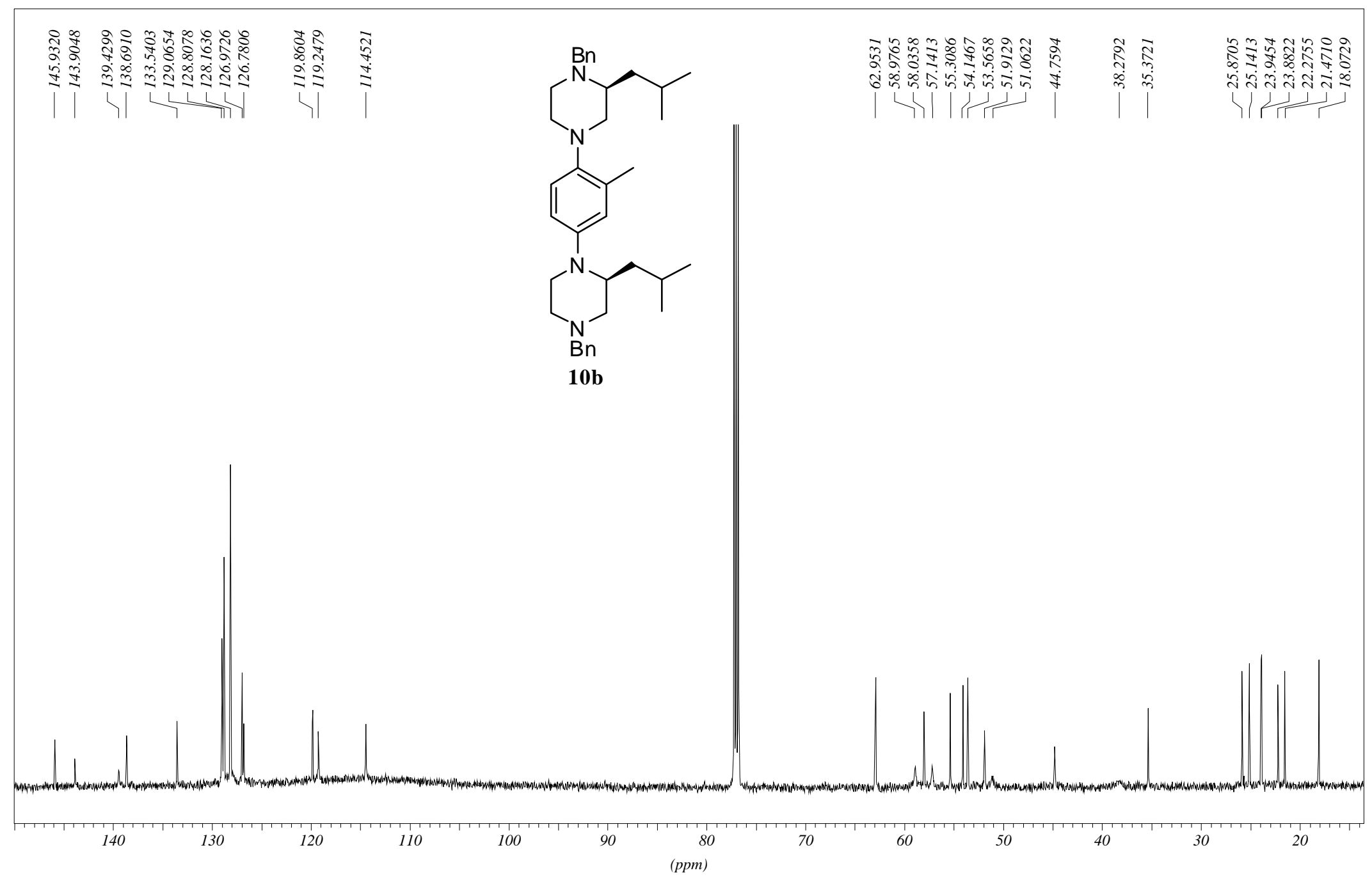




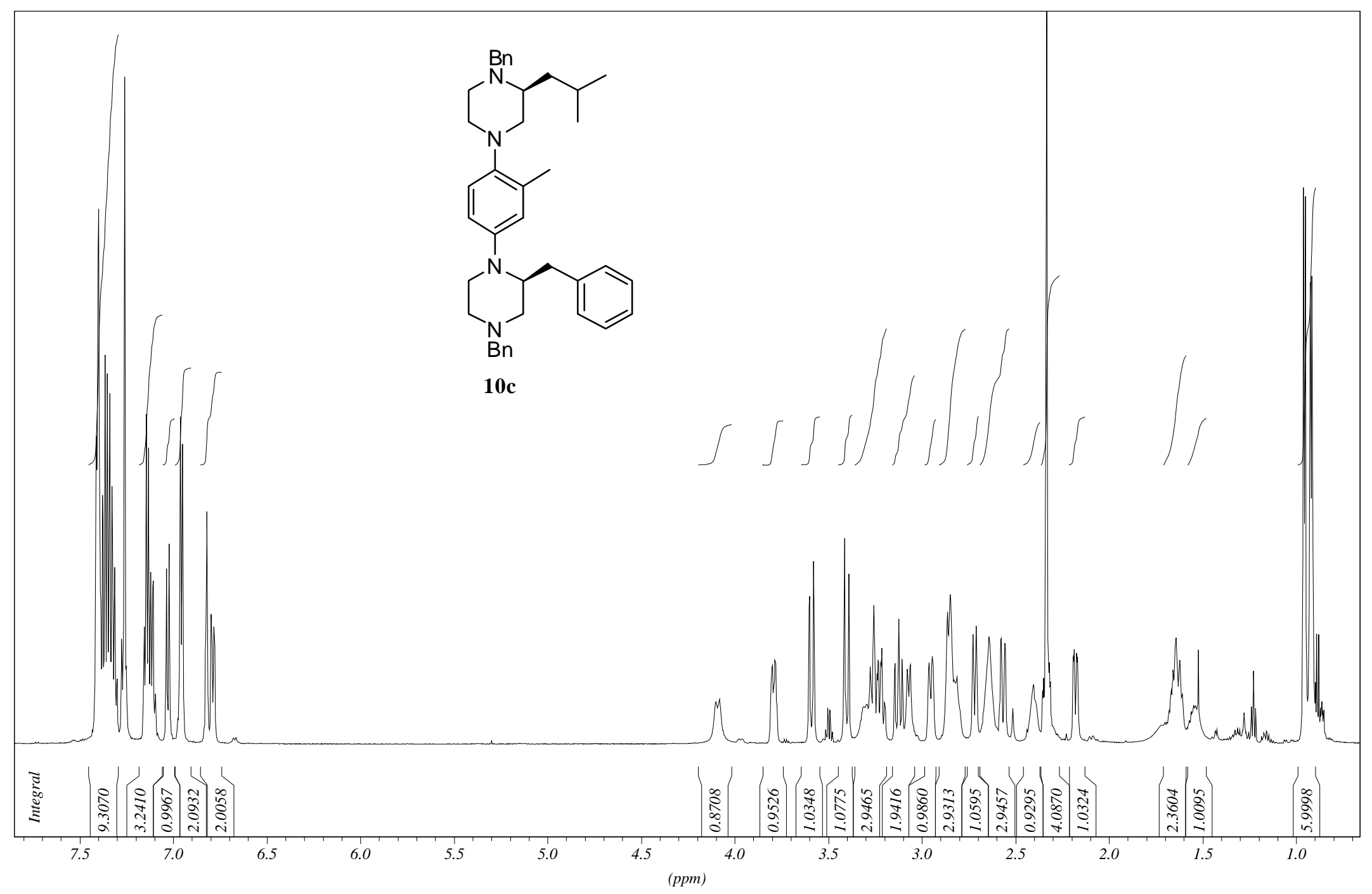




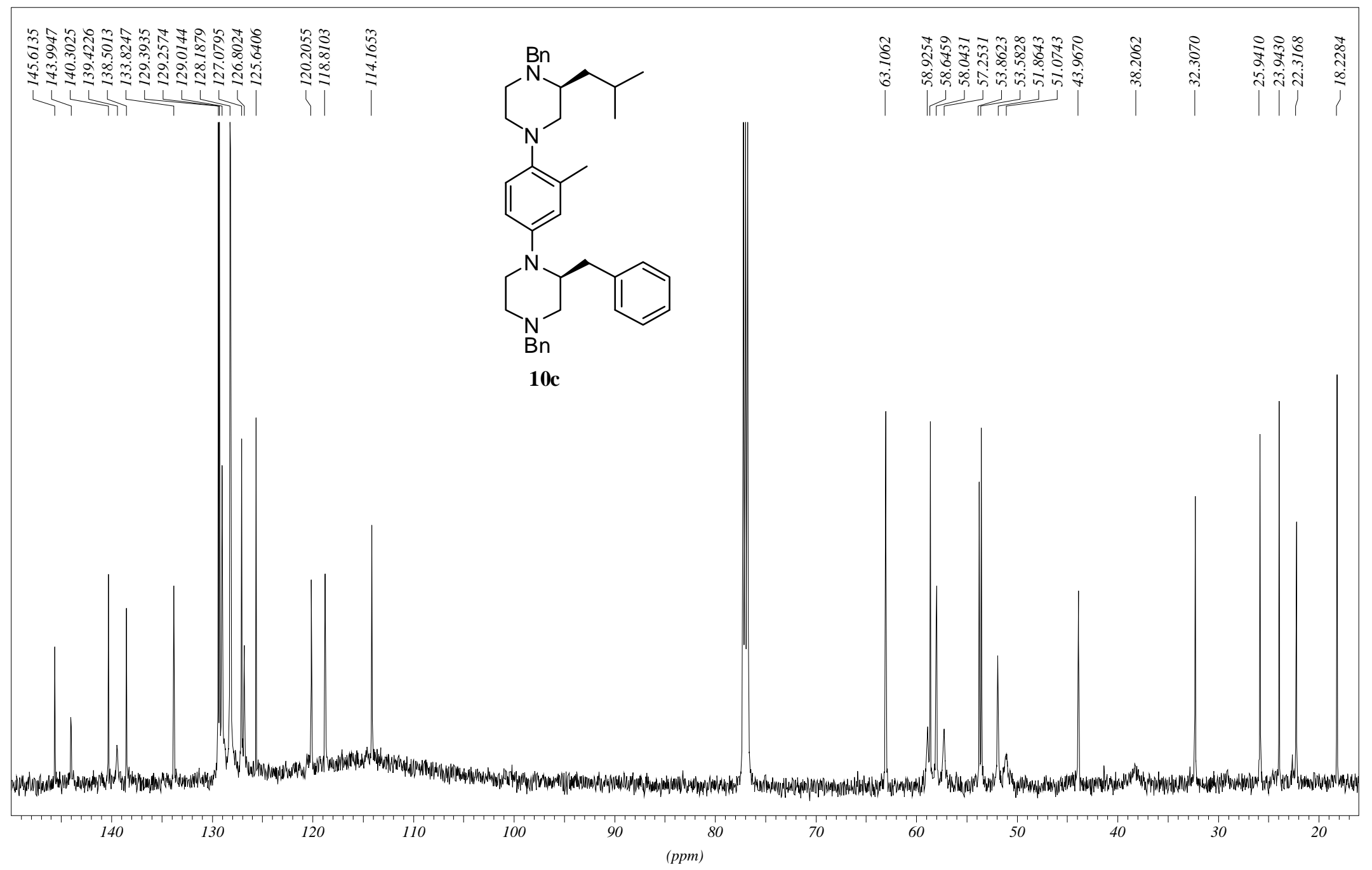

